\title{
Isolation improvement of compact LTE MIMO terminal using combination of neutralisation line and modified ground structure
}

\author{
HS Singh $^{1^{*}}$ and RM Shubair ${ }^{2}$ \\ ${ }^{1}$ Department of Electronics and Communication Engineering, Thapar Institute of Engineering and Technology, Patiala-147004, Punjab, India. \\ ${ }^{2}$ Research Laboratory of Electronics, Massachusetts Institute of Technology, Cambridge, MA, USA.
}

Abstract: A multi-wideband MIMO antenna is presented for LTE MIMO terminals. The proposed antenna covers LTE700, GSM1800, GSM1900, UMTS, LTE2500, and Wi-Fi frequency bands. The multi-antenna elements are placed at the top two corners of printed circuit board (PCB) in the mirror image position. Each antenna element consists of a driven element and a shorted radiating element. To make the compact antenna structure, radiating element is meandered, folded, and shorted to the ground through copper wire. The size of each radiator is $15 \times 25 \times 4 \mathrm{~mm}^{3}$. Further, a combination of neutralisation line and modified ground structure is designed and deployed between radiating elements to reduce the mutual coupling between them. According to the measurement results of the fabricated antenna prototype, the isolation between two antenna ports is better than $-12 \mathrm{~dB}$ over the operating frequency band. The simulated and measured results are found in close agreement. Moreover, to investigate the performance of the proposed antenna on the real application platform, mobile environment and user proximity analyses are carried out. Besides, total radiated power (TRP), channel capacity loss (CCL) and specific absorption rate (SAR) to peak location spacing ratio (SPLSR) are calculated and found well below the limit of Federal Communication Commission (FCC) standard.

Keywords: Isolation, LTE, MIMO antenna, neutralisation line, SAR.

\section{INTRODUCTION}

The long-term evolution (LTE) multiple-input and multiple-output (MIMO) systems have drawn an enormous attention due to their efficacy in mitigating the multipath fading effect and high channel capacity without sacrificing additional transmitted power (Murch \& Letaief, 2002). A lower envelope correlation of MIMO antennas is desirable to mitigate the multipath fading problem, which is achieved by high port-to-port isolation. Therefore, in a multi-element MIMO system, the spacing between the antenna elements should be larger than the half wavelength. Unfortunately, it is not acceptable in a mobile handset.

Over the last few years, many techniques are explicated to provide high isolation between the antenna elements, which can be placed into various categories such as ground decoupling (Coetzee \& Yu, 2008; Wong et al., 2011; Meshram et al., 2012; Rowell \& Lam, 2012; Singh et al., 2018), port decoupling (Coetzee \& Yu, 2008) and polarisation decoupling (Zhang et al., 2012). In the ground decoupling method, different geometrical structures are used to isolate the antenna ports while in the port decoupling method, feeding of orthogonal eigen modes to the antenna array takes place. In the port decoupling method, a modal feed network is connected to the antenna array, which provides additional complexity inside the handsets. In the polarisation decoupling method, orthogonally arranged antenna array is used which produces orthogonal radiation pattern, that results in low mutual coupling through the orthogonal modes of current on the system's ground. Nevertheless, for closely placed compact multi-wideband MIMO antenna, most of the available decoupling methods are not applicable

"Corresponding author (harishankar1990@gmail.com; (10) https://orcid.org/0000-0002-0125-688X) 
due to large area mounting, well-designed decoupling structure, and single band or narrow band operation. To the best of authors' knowledge, a combination of neutralisation line and modified ground structure is not used for isolation improvement in the open literature.

Moreover, pattern of mobile handsets are going towards slimness, multi-band operation and high transmit and receive performance. Hence, it is a challenge for antenna designers to implement multiple multi-band antennas within the confined space of mobile handset and avoid interaction with the user body. The antenna performances such as efficiency, radiation pattern, and impedance matching greatly depend on the nearby object, especially the presence of user body in close proximity of the antenna. Given the actual scenario limitation and requirement of low profile with good impedance matching over the multi-band is indeed challenging from the antenna design point of view. So far, many researchers have reported on studies of mobile terminal antennas in close proximity of the human body (Pelosi et al., 2010; Yu et al., 2010; Gao et al., 2013; Zhang et al., 2013; Singh et al., 2015). However, some of the studies are carried out over a single antenna element (Pelosi et al., 2010; Yu et al., 2010; Gao et al., 2013) while in Zhang et al. (2013), four elements are considered on the mobile phone printed circuit board (PCB) for adaptive analysis. Here, the placement of four elements within the confined space of mobile phone will provide complexity for other mobile circuitries. Recently, a MIMO antenna has been proposed and explicitly investigated in the actual scenario of a mobile phone (Singh et al., 2015). However, investigated antennas have $3 \mathrm{D}$ geometry structure which increases complexity within smart mobile handsets. The comparison of size, type of antenna, operating frequency bands, specific absorption rate (SAR) to peak location spacing ratio (SPLSR), efficiency, and gain of the proposed antenna with reference antennas are given in Table 1. It is interesting to note that the proposed antenna is showing optimal performance by trade-off between size, planarity of structure, operating bands, SPLSR and radiation performances.

In this paper, a compact multi-wideband MIMO antenna is proposed and it provides application platform in LTE700, GSM1800, GSM1900, UMTS, LTE2500, and Wi-Fi. To reduce the mutual coupling between inter-antenna elements, a combination of neutralisation line and modified ground structure is used. The surface current coupling through the ground plane is reduced using modified ground structure, whereas the space wave coupling is reduced by neutralisation line. Hence a combination of these two provides better isolation $(>12 \mathrm{~dB})$ over the interest of frequency. Further, mobile environment and user proximity analysis are carried out to check the robustness of the proposed antenna.

\section{METHODOLOGY}

The proposed MIMO antenna is designed on $0.8 \mathrm{~mm}$ thick FR4 substrate of relative permittivity $\left(\varepsilon_{\mathrm{r}}\right) 4.4$ and loss tangent $(\tan \delta) 0.018$. The whole size of the mobile phone PCB is $100 \times 60 \mathrm{~mm}^{2}$. Two radiating elements of the MIMO antenna are printed on the top two corners of the substrate with no ground portion underneath the radiating elements. The feed points and shorting points of the radiating elements are at $A_{1}$ and $A_{2}$ and $B_{1}$ and $\mathrm{B}_{2}$, respectively as shown in Figure 1(a). The feed point and shorting point of Element-1 with respect to origin is $\left(\mathrm{x}_{1}, \mathrm{y}_{1}=4.76 \mathrm{~mm}, 84.5 \mathrm{~mm}\right)$ and $\left(\mathrm{x}_{2}, \mathrm{y}_{1}=6.43 \mathrm{~mm}\right.$, $84.5 \mathrm{~mm})$, respectively, whereas Element-2 is the mirror image of Element-1. The front view and rear view (dimensions of modified ground plane) of the proposed antenna are depicted in Figure 1(a) and dimensions of the unfolded single antenna element are shown in Figure 1(b). The driven element is used to excite the folded shorted main radiating element. The design mechanism of the proposed antenna can be revealed by reflection coefficient plot as shown in Figure 2(a). Initially, driven element is designed having a length $\lambda / 4(\cong 30.3 \mathrm{~mm})$ at $2.5 \mathrm{GHz}$ and it resonates correspondingly as shown in Figure 2(a). In order to obtain lower and wideband upper-frequency band, a quarter wave shorting element is designed while the initially designed driven element is used as a coupling feed. The planar shorting strip resonates at $0.825 \mathrm{GHz}$ corresponding to the electrical length $\lambda / 4(\cong 91 \mathrm{~mm})$ at $0.825 \mathrm{GHz}$. The coupling between the driven element and the shorted planar strip provides wideband at upper frequency. However, lower frequency at $0.825 \mathrm{GHz}$ is achieved due to the flow of current (generated due to mutual coupling between driven element and shorted planar strip) from coupling point [as shown in Figure 2(b)] to end terminal of the planar element. Finally, to achieve LTE700 frequency band as well as to compact the antenna structure, a vertical strip is added to the planar element. Now, the total electrical length of the shorted planar element is increased to $\lambda / 4(\approx 98.6 \mathrm{~mm})$ at $0.76 \mathrm{GHz}$ and resonance is shifted from $0.825 \mathrm{GHz}$ to $0.76 \mathrm{GHz}$ corresponding to the quarter wavelength of the folded shorting strip.

\section{RESULTS AND DISCUSSION}

The combination of neutralisation line and modified ground plane plays a vital role in isolation enhancement and impedance matching. To analyse the principle of 
isolation technique, simulated surface current distribution on the antenna elements and system ground plane are plotted at $0.75 \mathrm{GHz}$ and $1.8 \mathrm{GHz}$ as shown in Figure 3(a) and the reflection coefficient is shown in Figure 3(b).

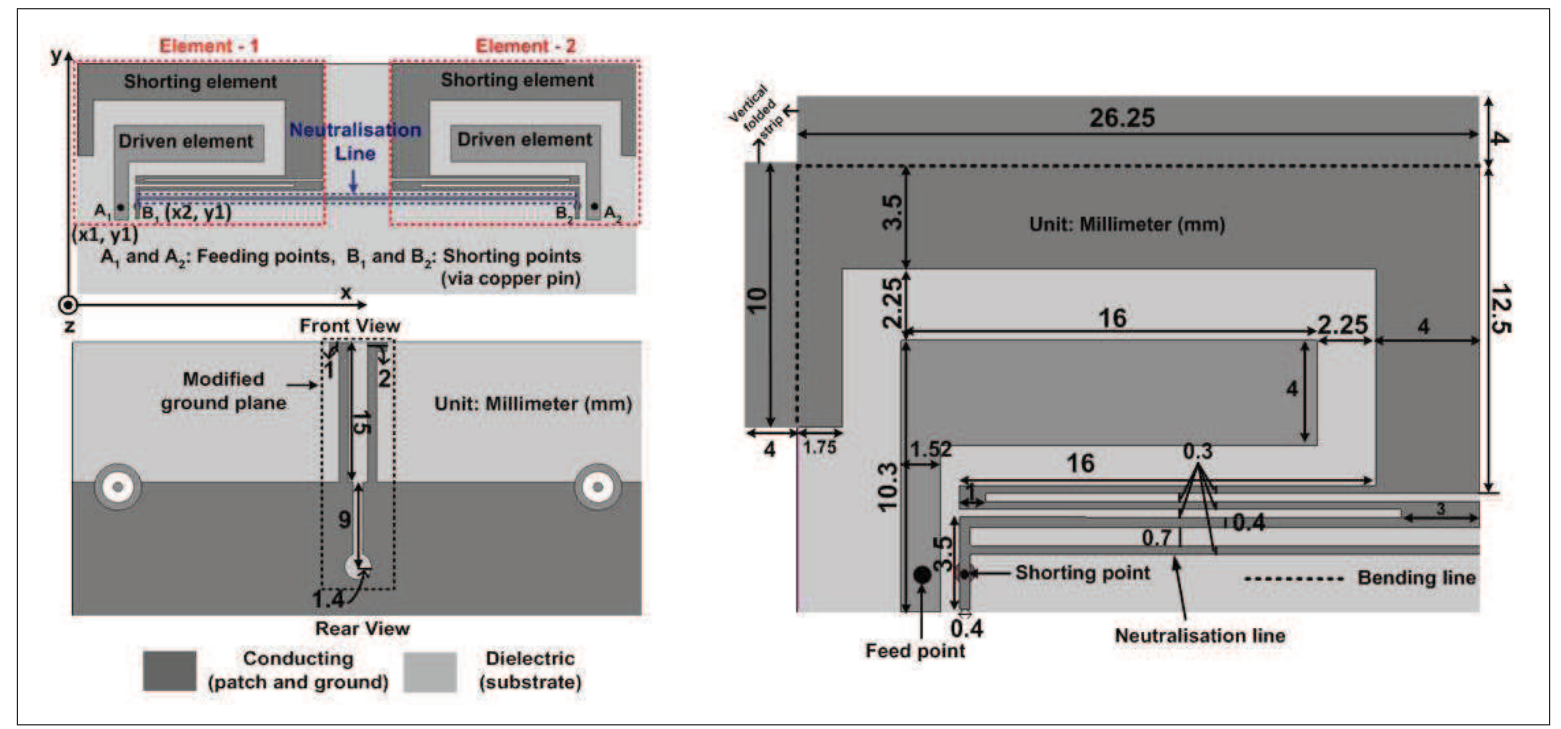

Figure 1: Configuration of the MIMO antenna: (a) Front and rear 2D view of the proposed antenna; (b) detail dimension of single antenna element

Table 1: Comparison of proposed antenna with reference antennas

\begin{tabular}{|c|c|c|c|c|c|}
\hline Reference antenna & $\begin{array}{l}\text { Size of the antenna } \\
\qquad\left(\mathrm{mm}^{3}\right)\end{array}$ & $\begin{array}{l}\text { Type of the } \\
\text { antenna }\end{array}$ & $\begin{array}{l}\text { Range of the frequency } \\
\text { (Operating bands) }\end{array}$ & SPLSR & Efficiency/gain \\
\hline $\begin{array}{l}\text { Meshram et al. } \\
\text { (2012) }\end{array}$ & $12 \times 20 \times 0.8$ & PIFA (3D) & $\begin{array}{c}\text { 746-787 MHz, } 1850-990 \mathrm{MHz} \text {, } \\
1920-2170 \mathrm{MHz} \text {, and } 3600-3700 \mathrm{MHz} \\
\text { (LTE \& Wi-Fi) }\end{array}$ & Not calculated & Not given \\
\hline $\begin{array}{l}\text { Rowell and Lam } \\
\text { (2012) }\end{array}$ & $35 \times 30 \times 10$ & PIFA (3D) & $\begin{array}{l}0.8-1 \mathrm{GHz} \text { and } 1.7-2.2 \mathrm{GHz} \\
(\mathrm{GSM}+3 \mathrm{G} / 4 \mathrm{G})\end{array}$ & Not calculated & $\begin{array}{c}77 \% \text { (average) } / \\
1-2 \mathrm{~dB}\end{array}$ \\
\hline Wong et al. (2011) & $\begin{array}{c}15 \times 30 \mathrm{~mm}^{2} \\
\text { (main) and } 21 \times 20 \\
\mathrm{~mm}^{2} \text { (auxiliary) }\end{array}$ & $\begin{array}{l}\text { Vertical } \\
\text { folded shorted } \\
\text { monopole } \\
\text { antenna }\end{array}$ & $\begin{array}{c}\text { 704-960/1710-2690 MHz (main) and } \\
\text { 704-787/2300-2690 MHz (auxiliary) } \\
\text { (LTE/ WWAN) }\end{array}$ & Not calculated & $\begin{array}{c}48-60 \%(\mathrm{LTE}) \\
\text { and } 63-82 \% \\
\text { (WWAN) }\end{array}$ \\
\hline Zhang et al. (2012) & $25 \times 40 \times 1.55$ & $\begin{array}{l}\text { Monopole } \\
\text { antenna }\end{array}$ & $\begin{array}{l}\text { 3.1-5.15 GHz } \\
\text { (UWB) }\end{array}$ & Not calculated & $\begin{array}{c}-2.5 \text { to }-1 \mathrm{~dB} / \\
>1.5 \mathrm{~dB}\end{array}$ \\
\hline Proposed & $15 \times 25 \times 4$ & $\begin{array}{l}\text { Shorted } \\
\text { monopole }\end{array}$ & $\begin{array}{l}\text { LTE700, GSM1800, GSM1900, UMTS, } \\
\text { LTE2500, and Wi-Fi }\end{array}$ & $<0.3$ & $\begin{array}{c}25-72 \% / 0.2-2.5 \\
\mathrm{~dB}\end{array}$ \\
\hline
\end{tabular}




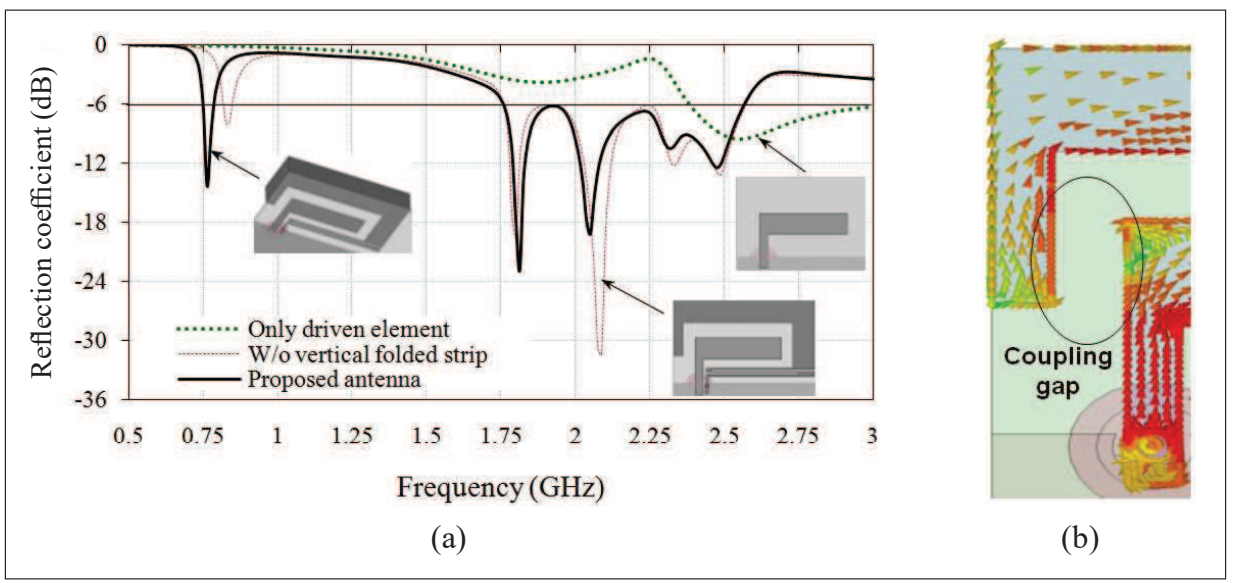

Figure 2: Operating mechanism: (a) reflection coefficient of the proposed antenna; (b) vector current to show coupling between the driven and planar strip

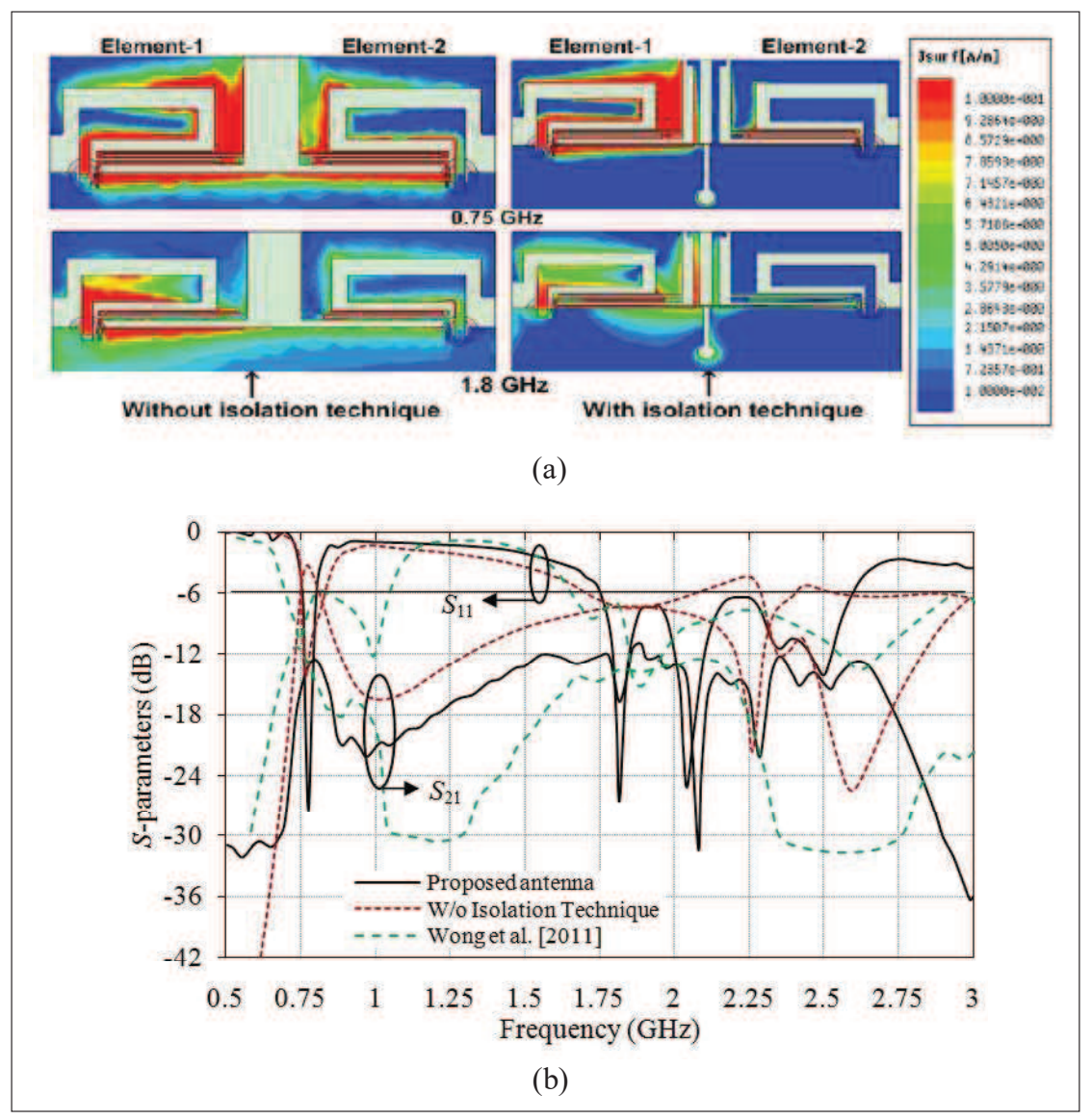

Figure 3: Isolation mechanism: (a) surface current distribution; (b) effect of isolation technique on $S$-parameters and comparison with reference antenna 
The proposed isolation technique changes the current distribution on the ground plane, neutralisation line, and antenna elements, whose current density is apparently reduced, providing enhanced isolation. For the lower band as well as the higher band, a combination of neutralisation line and modified ground plane generates a null point around antenna Element-2, which is beneficial in antenna array decoupling. Further, isolation of the proposed antenna was also compared with the reference antenna (Wong et al., 2011). It is noticed that the proposed isolation technique provides better isolation enhancement as compared to the technique applied by Wong et al. (2011). Further, size of the antenna is less than that of the reference antenna.

Finally, the proposed antenna was fabricated and measured by Anritsu made VNA Master MS2038C $(5 \mathrm{kHz}-20 \mathrm{GHz})$. The fabricated antenna and measured results are shown in Figure 4(a) and Figure 4(b),
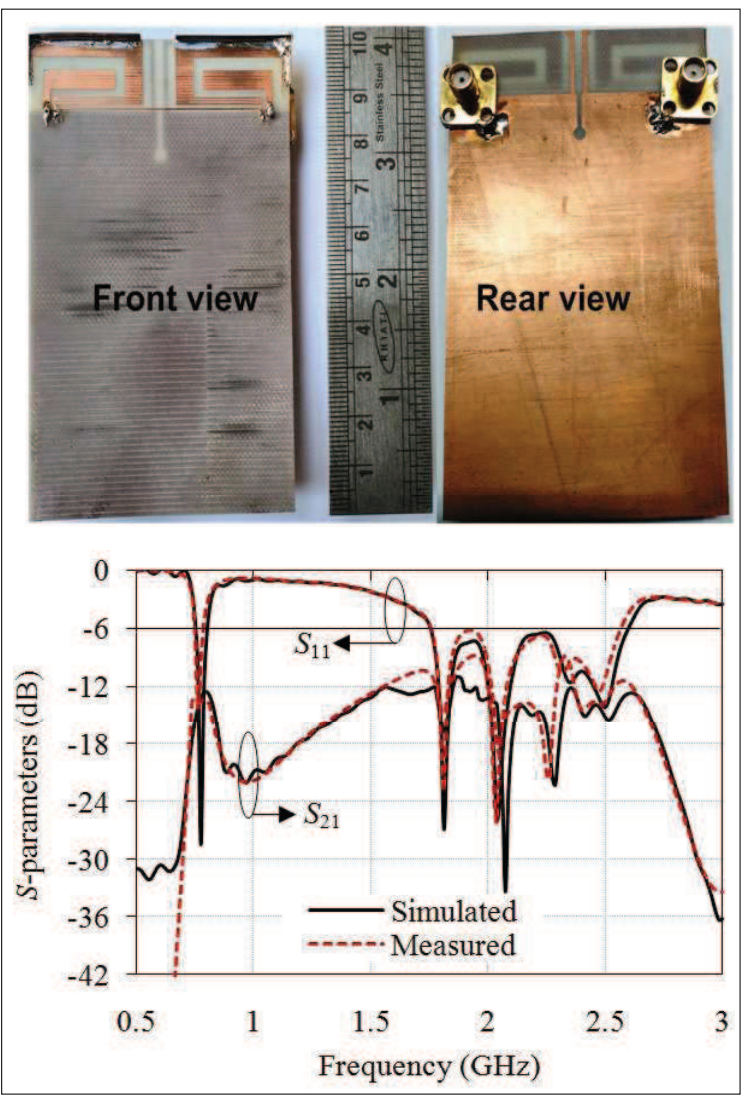

Figure 4: Antenna fabrication and measurement: (a) fabricated prototype; (b) simulated and measured $S$-parameters respectively. The simulated and measured results are well matched. Some discrepancies were observed due to manufacturing and measurement tolerances.

Figure 5 depicts the simulated and measured radiation patterns of the proposed MIMO antenna. The simulated $3 \mathrm{D}$ radiation patterns of Element-1 and Element-2 at $0.75 \mathrm{GHz}$ and $1.8 \mathrm{GHz}$ are shown in Figure 5(a). The 3D simulated radiation patterns of the dual elements antenna array point to the complimentary spatial region, which shows pattern diversity of the proposed antenna and this property can provide low correlation and good antenna diversity in free space. Further, to confirm the pattern diversity of the proposed MIMO antenna, simulated and measured 2D radiation patterns are shown in Figure 5 (b). It is interestingly noted that the measured radiation patterns provide pattern diversity with almost equal gain as found in the simulated 3D patterns. However, the simulated and measured results are well matched. Some discrepancies appeared due to manufacturing and measurement tolerances.

The calculated total radiation efficiency by considering the reflection loss and measured gain are shown in Figure 6. It is observed that the total antenna efficiency varies over the operating frequency between 10 to $75 \%$. The calculated total efficiency at $0.77,1.8$, 2.1 and $2.5 \mathrm{GHz}$ are 30, 54, 67 and $72 \%$, respectively while the variation of measured gain lies between 0.2 to $2.5 \mathrm{dBi}$ over the operating frequency range.

\section{Actual mobile environment and user proximity analysis}

Study of the proposed MIMO antenna in actual scenario (mobile environment and user proximity) is equally important to check the robustness on the actual platform. A smart phone has a number of components besides system circuit board. The typical mobile phone configuration with the proposed antenna is shown in Figure 7(a). There is a camera (diameter: $6 \mathrm{~mm}$ and thickness: $5 \mathrm{~mm}$ ) placed just behind and centre of the MIMO antenna elements. At the top of the PCB, a speaker with volume of $5 \times 1$ $\times 2 \mathrm{~mm}^{3}$ is placed. A large-size touch screen LCD with volume of $73 \times 55 \times 2 \mathrm{~mm}^{3}$ and a battery with volume of $66 \times 48 \times 3 \mathrm{~mm}^{3}$ are located parallel with a spacing of $1 \mathrm{~mm}$ from the circuit board and are connected to the main circuit board via connectors. All these components are assumed to be perfect electric conductors (PECs) for the simulation. One microphone is also accounted, and it is located far from the antenna elements. A plastic box, which has a relative permittivity of 3 , loss tangent of 0.06 , and conductivity of $0.24 \mathrm{~S} / \mathrm{m}$ is assumed to form 
the housing of the mobile phone. The simulation setup of mobile environment is created in finite integration technique (FIT) based computer simulation technology microwave studio (CST MWS).

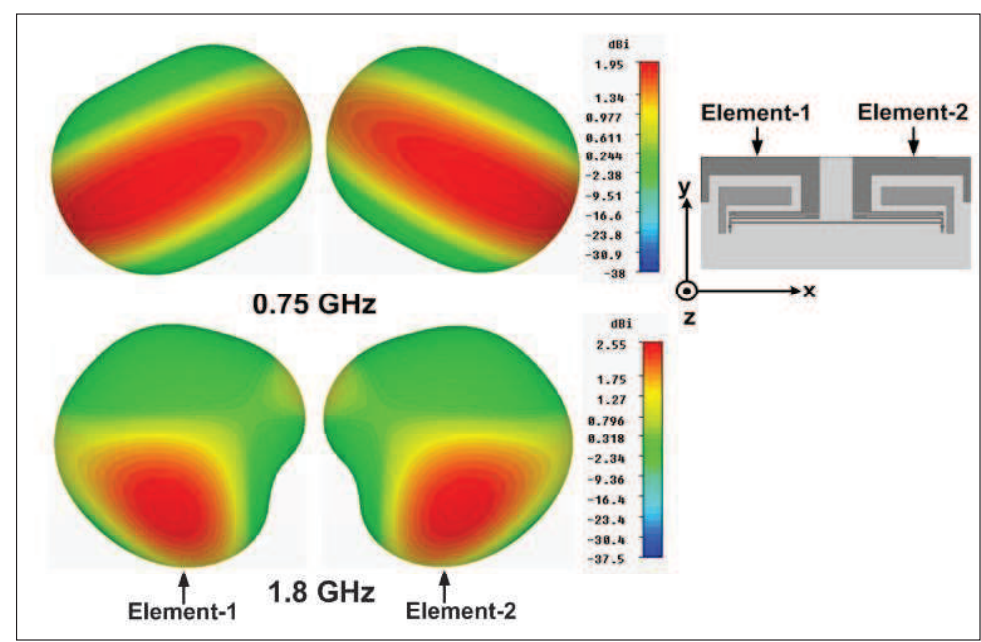

(a)

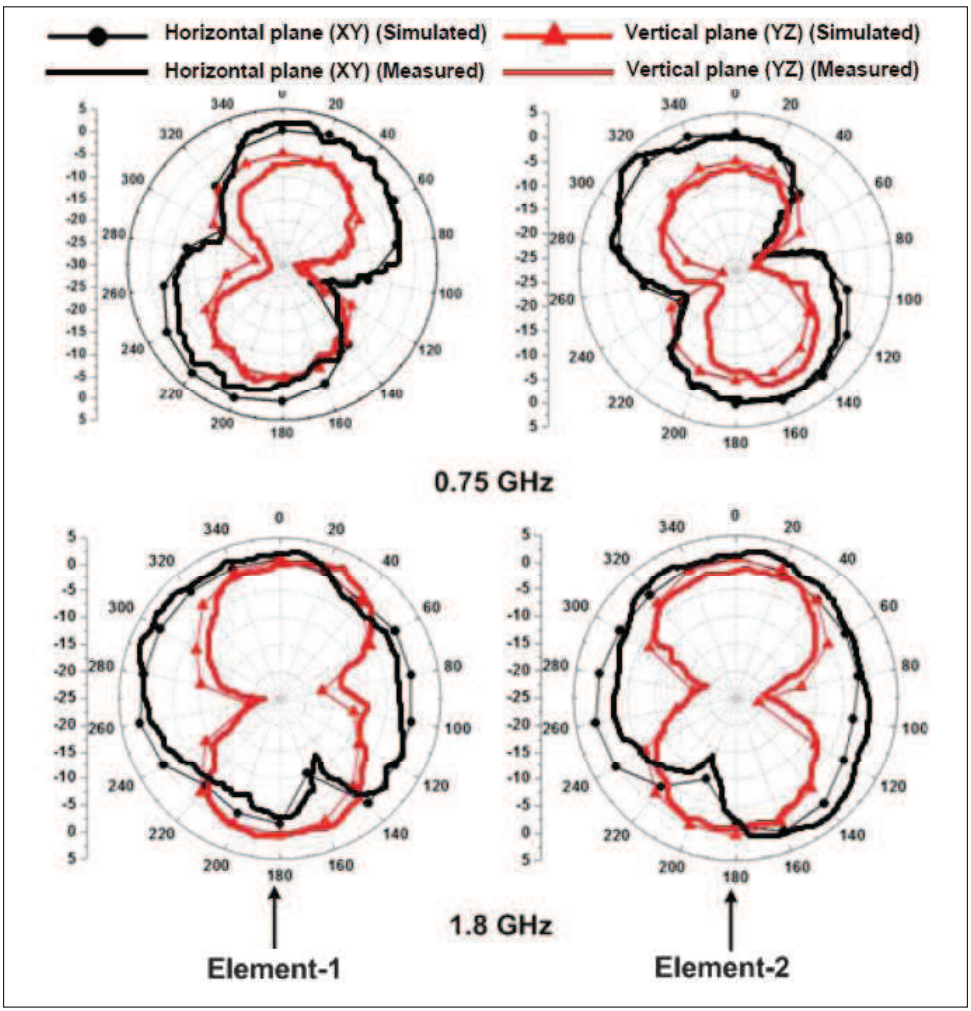

(b)

Figure 5: Radiation patterns of the proposed antenna at $0.77 \mathrm{GHz}$ and $1.8 \mathrm{GHz}$ : (a) simulated 3D radiation patterns; (b) simulated and measured radiation patterns 


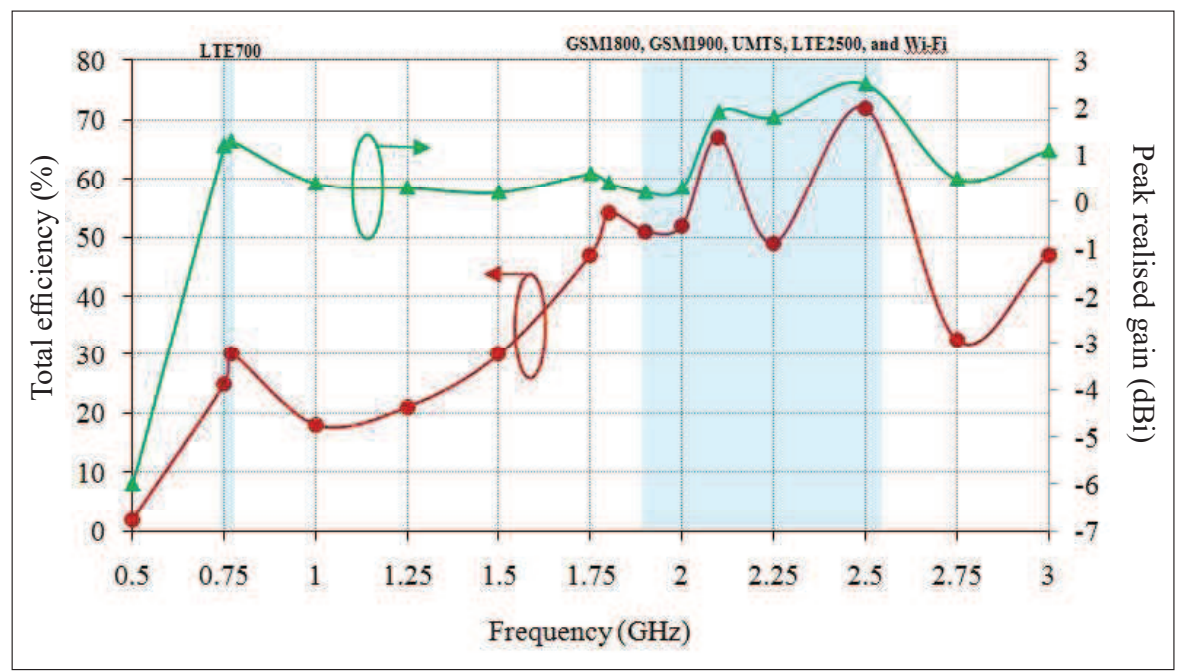

Figure 6: Variation of total radiation efficiency and peak realised gain

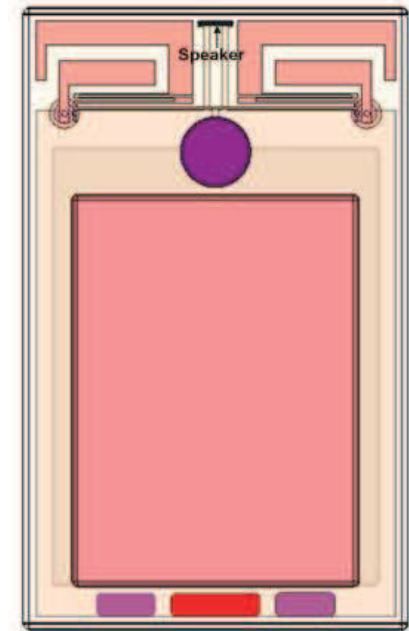

Front view

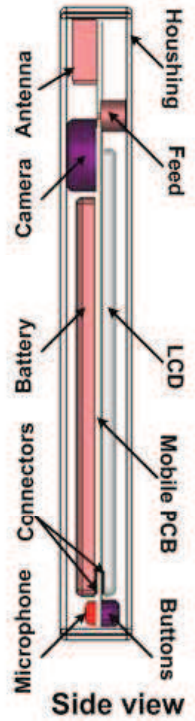

(a)

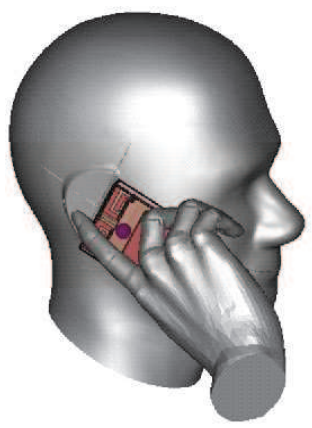

(b)

Figure 7: (a) Typical mobile phone configuration with proposed antenna; (b) talk mode (SAM head and PDA hand) with mobile phone configuration

Further, the mobile phone (antenna with mobile environment) is placed close to the human head in accordance with the cellular telecommunication industry association (CTIA) revision 3.1 (CTIA, 2011). The human head model consists of two layers, namely, fluid and shell. Fluid is confined in the shell, which is the outer layer. The dielectric properties of the fluid and shell are taken from CTIA (2011). Generally, mobile phone users use their mobile phone in the talk mode [specific anthropomorphic mannequin (SAM) head and personal 
digital assistants (PDA) hand] (Singh et al., 2019; Singh $\&$ Subair, 2019). As each element is placed symmetrical on the mobile phone PCB, it does not make any difference while using the left or right hand. Therefore, use of the left or right hand will not cause any difference. In the simulation set up, it is assumed that the right hand is used (as most people do). The simulation set up of the mobile phone in the user proximity is shown in Figure 7(b).

\section{$S$-parameter analysis}

In order to check the robustness of the proposed MIMO antenna performance with user proximity, initially $S$-parameters are studied. The variation of the $S$-parameters in free space, mobile environment, and user proximity [talk mode (SAM head and PDA hand)] are shown in Figure 8. It is of interest to note that the impedance matching at LTE700 frequency band is slightly getting disturbed in the presence of mobile environment and user proximity but still covers the desired LTE700 (0.747 GHz to $0.787 \mathrm{GHz})$ operating frequency band based on $-6 \mathrm{~dB}$ reflection coefficient. At the higher operating frequency band, the bandwidth is slightly increased in the presence of user proximity and covers the whole WWAN $(1.75 \mathrm{GHz}$ to $2.57 \mathrm{GHz})$ operating frequency in the presence of mobile as well as user environment based on $-6 \mathrm{~dB}$ reflection coefficient. Moreover, the isolation between the antenna elements is well below $-10 \mathrm{~dB}$ in all the cases.

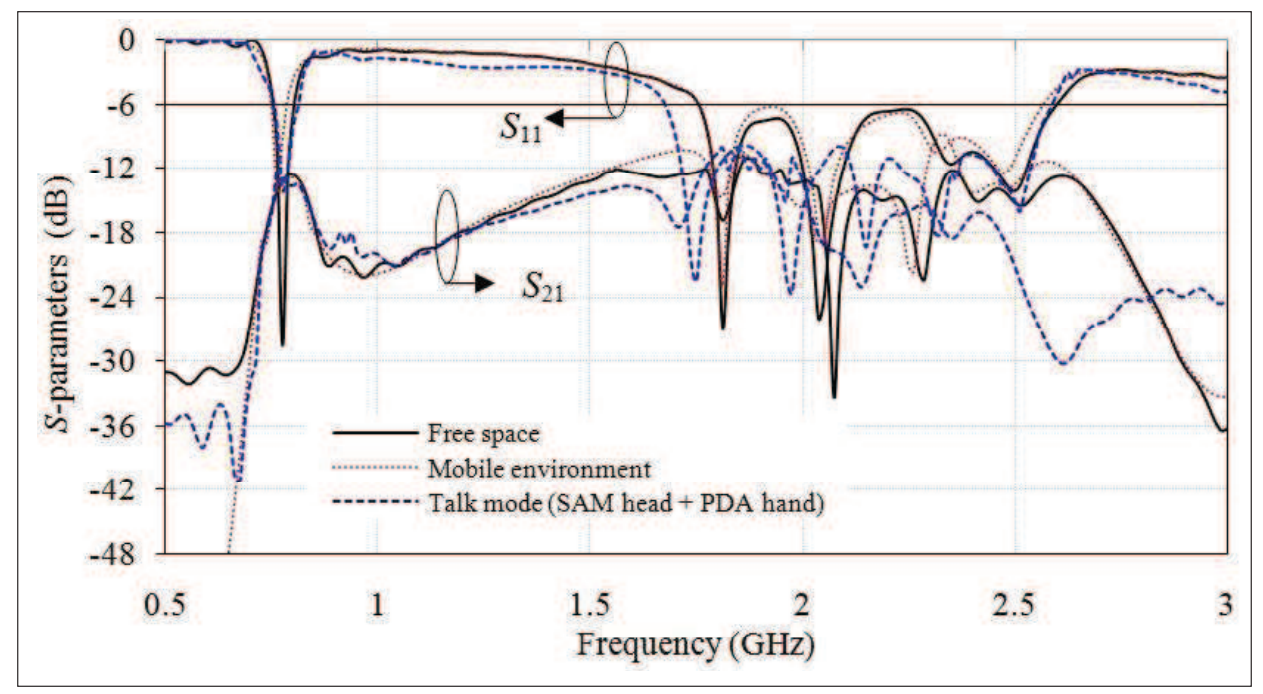

Figure 8: Variation of $S$-parameters in free space, mobile environment, and user proximity

\section{Diversity performances analysis}

The envelope correlation coefficient (ECC) and mean effective gain (MEG) parameters are investigated for evaluating the diversity capability of the proposed MIMO antenna. Calculation of the diversity parameters is carried out using CST MWS. The ECC results are calculated through $3 \mathrm{D}$ far field radiation patterns, as given in equation (1). In which, the envelope correlation coefficient $\left(\rho_{\mathrm{e}}\right)$ is given in terms of complex cross correlation $\left(\rho_{\mathrm{c}}\right)$

$\rho_{e} \approx\left|\rho_{c}\right|^{2}$
The $\rho_{\mathrm{c}}$ is evaluated using radiation pattern as given by Vaughan and Andersen (1987),

$$
\rho_{e i j}=\frac{\int_{0}^{2 \pi} \int_{0}^{\pi} A_{i j}(\theta, \phi) \sin \theta d \theta d \phi}{\sqrt{\int_{0}^{2 \pi} \int_{0}^{\pi} A_{i i}(\theta, \phi) \sin \theta d \theta d \phi} \sqrt{\int_{0}^{2 \pi} \int_{0}^{\pi} A_{j j}(\theta, \phi) \sin \theta d \theta d \phi}}
$$

where,

$$
\begin{aligned}
A_{i j}=X P R * E_{\theta, i}(\theta, \phi) E_{\theta, j}^{*}(\theta, \phi) P_{\theta}(\theta, \phi)+ \\
E_{\phi, i}(\theta, \phi) E_{\phi, j}^{*}(\theta, \phi) P_{\phi}(\theta, \phi)
\end{aligned}
$$


where, $E_{\theta, \phi}(\theta, \phi)$ and $P_{\theta, \phi}(\theta, \phi)$ are respectively the complex electric field and power angular density function of the incoming plane waves in the $\theta$ - and $\phi$ polarizations. $X P R$ is the vertical to horizontal average power ratio. Where $i, j=1,2$ and $i \neq j$.

Further, MEG parameter is considered for the study. MEG gives the statistical measure of the antenna gain in a given mobile environment. The MEG is defined as the ratio between the mean received power of antennas over a random route and the total mean incident power at the antenna elements. In order to calculate the MEG of a particular antenna element, the following expression given in equation (2) is used (Taga, 1990):

$$
\begin{aligned}
M E G=\int_{0}^{2 \pi} \int_{0}^{\pi}\left[\frac{X P R}{1+X P R} G_{\theta}(\theta, \phi) P_{\theta}(\theta, \phi)+\right. \\
\left.\frac{1}{1+X P R} G_{\phi}(\theta, \phi) P_{\phi}(\theta, \phi)\right] \sin \theta d \theta d \phi
\end{aligned}
$$

where, $X P R$ is the vertical to horizontal average power ratio, $G_{\theta}$ and $G_{\phi}$ are the power gain patterns and $P_{\theta}$ and $P_{\phi}$ are the $\theta$ - and $\phi$ - components of angular density functions of the incident power, respectively. It is quite natural to assume that when a mobile user moves randomly in any environment, the incident waves can arise from any azimuth direction with equal probability. However, such assumption cannot be made in elevation direction. A few models have been presented in Taga (1990) and Pedersen et al. (1997). The distributions of angular density functions depend on the surrounding environment. Here, $P_{\theta}$ and $P_{\phi}$ are assumed to be Gaussian in elevation and uniform in azimuth, and are given in Taga (1990).

The calculated values of ECC and MEG in free space, mobile environment, and user vicinity are given in Table 2. It is noted that ECCs are well within the allowable limit of 0.5 in all the cases. Further, MEG1 and MEG2 are calculated for Element-1 and Element-2, respectively for the above mentioned environment and are given in Table 2. It is observed that the slight difference between MEG1 and MEG2, considering the fact that the two antennas are identical, is due to the difference in gain patterns at the assumed angle for the incident power. However, the ratio of MEGs is nearly close to unity, which satisfies the equality criterion for the MIMO antenna.

Table 2: Diversity parameters estimation in free space, mobile environment, and user proximity

\begin{tabular}{lccccc}
\hline Antenna proximity & Frequency $(\mathrm{GHz})$ & ECCs & MEG1 & MEG2 & MEG1/MEG2 \\
\hline Free space & 0.75 & 0.01 & -7.2 & -7.1 & 1.01 \\
& 1.8 & 0.0013 & -6.4 & -6.48 & 0.99 \\
Mobile environment & 2.1 & 0.002 & -5.6 & -5.8 & 0.96 \\
& 0.75 & 0.02 & -10.6 & -10.8 & 0.098 \\
User proximity & 1.8 & 0.0018 & -9.3 & -9.36 & 0.99 \\
(Talk mode) & 2.1 & 0.019 & -8.1 & -8.09 & 1.001 \\
& 0.75 & 0.028 & -17.72 & -17.79 & 0.99 \\
& 1.8 & 0.003 & -15.63 & -15.6 & 1.002 \\
\hline
\end{tabular}

\section{Channel capacity loss (CCL)}

Another important parameter is channel capacity loss (CCL), which is employed in the present work to characterise the equality of a multi antenna array. Thus, CCL is also investigated in free space and user proximity for top and bottom position antenna array. CCL of the antenna is estimated using $S$-parameter (Choukiker et al., 2014) and is given by equation (3):

$$
C_{\text {loss }}=-\log _{2} \operatorname{det}\left(\psi_{R}\right)
$$

where, $\psi_{R}$ is the receiving antenna correlation matrix. The matrix elements $\rho_{\mathrm{ij}}$ accounts for the amount of correlation. The expression shows MIMO system's performance and $C_{\text {loss }}$ is affected by the reflections at the antenna ports.

$$
\psi_{R}=\left(\begin{array}{ll}
\rho_{11} & \rho_{12} \\
\rho_{21} & \rho_{22}
\end{array}\right)
$$

$$
\begin{aligned}
& \text { Here, } \\
& \begin{array}{l}
\rho_{i i}=1-\left|S_{i i}\right|^{2}-\left|S_{i j}\right|^{2} \\
\rho_{i j}=-\left(S_{i i}^{*} * S_{i j}+S_{j i}^{*} * S_{i j}\right), \text { for } i, j=1 \text { or } 2
\end{array}
\end{aligned}
$$


The calculated values of CCL for multi antenna array in free space and user proximity are given in Table 3 . In free space, the measured CCL is in close agreement with the simulated one. Moreover, the calculated CCL in user proximity provides high value for bottom placed antenna in comparison to the top placed antenna array; this is due to the high correlation between multi antenna arrays. For a better MIMO antenna performance, CCL should be less than $0.4 \mathrm{bits} / \mathrm{s} / \mathrm{Hz}$. It is depicted from Table 3 that the values fall well below the level of $0.4 \mathrm{bits} / \mathrm{s} / \mathrm{Hz}$ in free space as well as in user proximity.

\section{TRP and SAR analysis}

TRP and SAR are considered to evaluate the performance of the proposed antenna on the actual mobile phone platform. The TRP correlates with the field performance of the antenna, which is influenced by the transmission power and the antenna efficiency. In this study, the conducted power is normalised to $1 \mathrm{~W}$ or $30 \mathrm{dBm}$. The TRP can be defined as follows (CTIA, 2005):

$$
\begin{aligned}
& \operatorname{TRP}=\int_{\theta=0}^{\pi} \int_{\phi=0}^{2 \pi} \operatorname{EiRP}(\theta, \phi) \sin \theta d \theta d \phi \\
& \operatorname{EiRP}(\theta, \phi)=P_{T} \cdot G_{T}(\theta, \phi)
\end{aligned}
$$

where, $P_{T}$ and $G_{T}$ are the power delivered to the antenna and the antenna gain, respectively.

\begin{tabular}{|c|c|c|c|c|}
\hline \multirow{3}{*}{$\begin{array}{c}\begin{array}{c}\text { Frequency } \\
(\mathrm{GHz})\end{array} \\
0.75\end{array}$} & \multicolumn{2}{|c|}{ Users condition } & \multirow{2}{*}{$\begin{array}{c}\begin{array}{c}\text { Antenna at } \\
\text { top }\end{array} \\
0.38\end{array}$} & \multirow{2}{*}{$\begin{array}{c}\begin{array}{c}\text { Antenna at } \\
\text { bottom }\end{array} \\
0.21\end{array}$} \\
\hline & \multirow{2}{*}{ Free space } & Simulated & & \\
\hline & & Measured & 0.37 & 0.19 \\
\hline & Talk mode & & 0.33 & 0.20 \\
\hline & Data mode & & 0.29 & 0.18 \\
\hline & Read mode & & 0.25 & 0.15 \\
\hline \multirow[t]{5}{*}{1.8} & \multirow{2}{*}{ Free space } & Simulated & 0.21 & 0.21 \\
\hline & & Measured & 0.29 & 0.25 \\
\hline & Talk mode & & 0.26 & 0.19 \\
\hline & Data mode & & 0.16 & 0.10 \\
\hline & Read mode & & 0.19 & 0.13 \\
\hline \multirow[t]{5}{*}{2.1} & \multirow{2}{*}{ Free space } & Simulated & 0.21 & 0.18 \\
\hline & & Measured & 0.22 & 0.21 \\
\hline & Talk mode & & 0.14 & 0.11 \\
\hline & Data mode & & 0.12 & 0.12 \\
\hline & Read mode & & 0.13 & 0.15 \\
\hline
\end{tabular}

Table 3: Variation of channel capacity loss
In addition, simplified formula of TRP can be defined approximately as given in equation (5),

$T R P(W)=$ Conducted Power $(W) \times E_{\text {miss }} \times E_{\text {rad }}$

where, $E_{\text {miss }}$ and $E_{\text {rad }}$ are the mismatch and the radiation efficiency of antenna, respectively and the conducted power is the transmitted power. In the case of dual element antenna array, TRP of elements are noted as TRP1 and TRP2 for Element-1 and Element-2, respectively. The calculated values of the TRP in free space, mobile environment, and user proximity are given in Table 4. It is observed that in free space and mobile environment, the TRP of both elements are same due to the uniform environment around each antenna element. However, the TRP in free space is slightly higher than the mobile environment due to low reflection losses and high total antenna efficiency. Further, the implementation of the proposed antenna along with mobile environment in user proximity provides lower TRP than free space and mobile environment due to the large human body around the mobile phone. However, the TRP in user proximity is still higher than $18 \mathrm{dBm}$. The higher TRP can significantly improve the call performance of the handset in a weak signal area.

Further, radiation from the antenna inside the human body can be evaluated by SAR, which is defined as the time rate of microwave energy absorbed inside the tissues, as given in equation (6) (CTIA, 2011).

$$
S A R=\frac{\sigma}{2 \rho} E^{2}
$$

where, $\sigma$ and $\rho$ are electrical conductivity $\left(\mathrm{kg} / \mathrm{m}^{3}\right)$ and density $(\mathrm{S} / \mathrm{m})$, respectively and $E$ is induced internal electrical field $(\mathrm{V} / \mathrm{m})$.

However, with the existing equipments, it is hard to measure the total SAR inside the human tissues when multi antenna elements operate simultaneously. In such scenario, SAR to peak location spacing ratio (SPLSR) is utilised to evaluate the SAR performance (FCC, 2008), as give in equation (7);

$S P L S R=\left(S A R_{1}+S A R_{2}\right) / D$

where, $S A R_{1}$ and $S A R_{2}$ are the average values of SAR (W/ $\mathrm{kg}$ ) for the antenna Elements 1 and 2, respectively, over human head tissue based on the FCC standard. $D$ is the separation distance $(\mathrm{cm})$ of the two SAR peaks. From 
the safety point of view, the SPLSR is required to be less than 0.3 when the separation between the dual elements is less than $5 \mathrm{~cm}$ from the FCC standard.

The simulation set up is created in CST MWS to calculate the SPLSR. The separation distance of the two SAR peaks and the values of SARs of each antenna element are calculated using CST MWS simulation software. The distribution of electromagnetic energy inside the human head over $1 \mathrm{~g}$ and $10 \mathrm{~g}$ average tissue due to antenna Element-1 and Element-2 at 0.77, 1.8 and 2.1 GHz are shown in Figure 9. The calculated values of SPLSR over $1 \mathrm{~g}$ and $10 \mathrm{~g}$ average human tissue at the frequencies $0.77,1.8$ and $2.1 \mathrm{GHz}$ are given in Table 5. It is observed that the calculated values of
SPLSR are well below the defined limit by FCC, i.e. 0.3

Table 4: Calculated values of TRP in free space, mobile environment, and user proximity

\begin{tabular}{ccccccc}
\hline $\begin{array}{c}\text { Freq. } \\
(\mathrm{GHz})\end{array}$ & Free space & \multicolumn{2}{c}{$\begin{array}{c}\text { Mobile } \\
\text { environment }\end{array}$} & User proximity \\
& $\begin{array}{r}\text { TRP1 } \\
(\mathrm{dBm})\end{array}$ & $\begin{array}{r}\mathrm{TRP} 2 \\
(\mathrm{dBm})\end{array}$ & $\begin{array}{c}\mathrm{TRP} 1 \\
(\mathrm{dBm})\end{array}$ & $\begin{array}{r}\mathrm{TRP} 2 \\
(\mathrm{dBm})\end{array}$ & $\begin{array}{r}\text { TRP1 } \\
(\mathrm{dBm})\end{array}$ & $\begin{array}{r}\text { TRP1 } \\
(\mathrm{dBm})\end{array}$ \\
\hline 0.77 & 23.4 & 23.4 & 20.1 & 20.1 & 18.5 & 18.2 \\
1.8 & 24.3 & 24.3 & 24.2 & 24.2 & 19.2 & 19 \\
2.1 & 25.2 & 25.2 & 25 & 25 & 19.6 & 19.2 \\
\hline
\end{tabular}

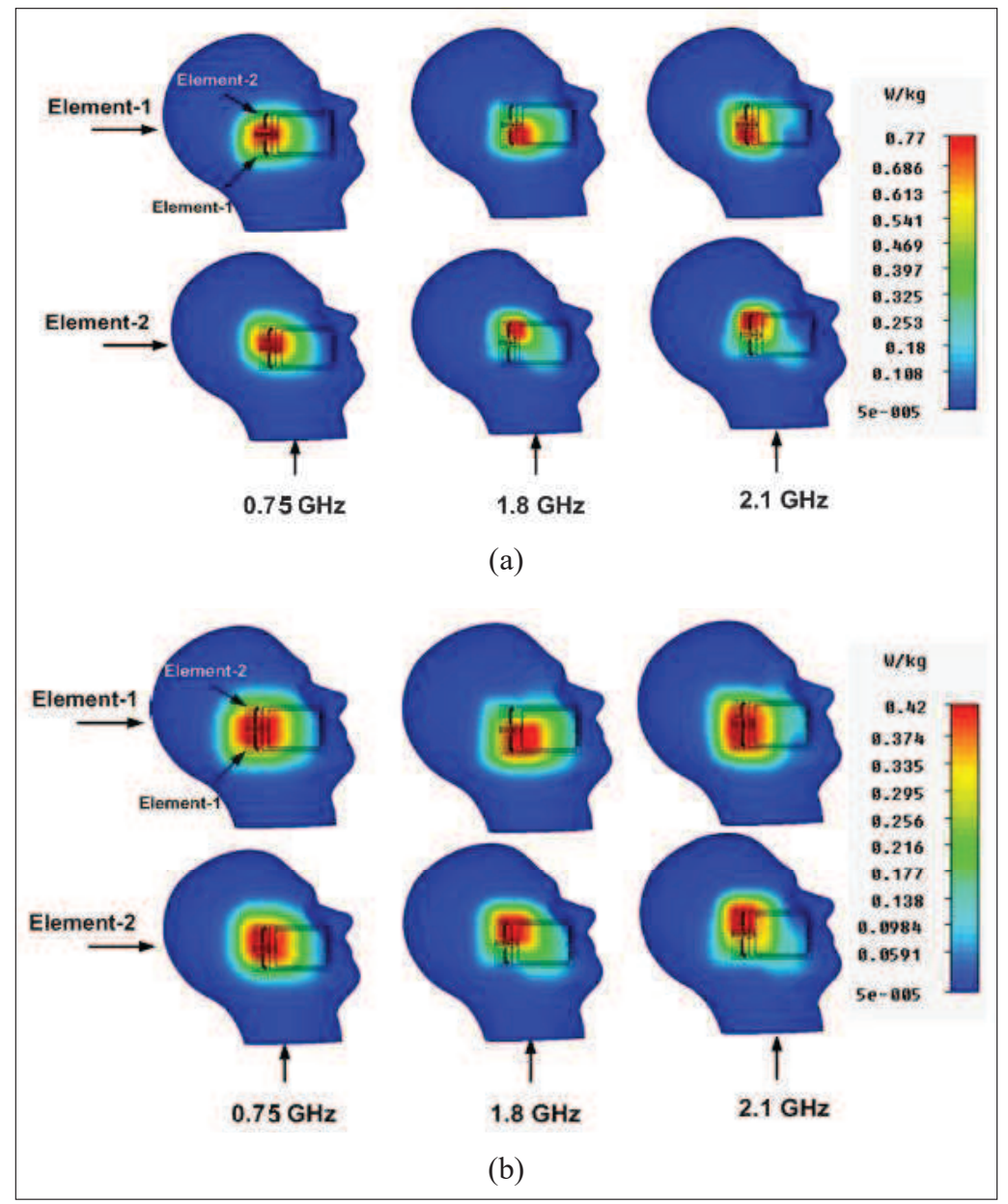

Figure 9: Distribution of energy over human tissue: (a) average SAR over $1 \mathrm{~g}$; (b) average SAR over $10 \mathrm{~g}$ 
Table 5: SPLSR performances of MIMO antenna in user proximity

\begin{tabular}{|c|c|c|c|c|c|c|c|c|}
\hline \multirow[b]{2}{*}{$\begin{array}{l}\text { Freq. } \\
(\mathrm{GHz})\end{array}$} & \multicolumn{4}{|c|}{ Distribution of SAR over $1 \mathrm{~g}$ tissue } & \multicolumn{4}{|c|}{ Distribution of SAR over $10 \mathrm{~g}$ tissue } \\
\hline & $\begin{array}{l}S A R_{l} \\
(\mathrm{~W} / \mathrm{kg})\end{array}$ & $\begin{array}{l}S_{2} R_{2} \\
(\mathrm{~W} / \mathrm{kg})\end{array}$ & $\begin{array}{l}\text { Separation } \\
\text { distance } b / \mathrm{w} \\
\text { SAR peaks } \\
\text { (D) }(\mathrm{cm})\end{array}$ & $\begin{array}{l}\text { SPLSR } \\
=\frac{\left(S A R_{1}+S A R_{2}\right)}{D} \\
(\mathrm{~W} / \mathrm{kg} / \mathrm{cm})\end{array}$ & $\begin{array}{l}S A R_{l} \\
(\mathrm{~W} / \mathrm{kg})\end{array}$ & $\begin{array}{l}S A R_{2} \\
(\mathrm{~W} / \mathrm{kg})\end{array}$ & $\begin{array}{c}\text { Separation } \\
\text { distance } \mathrm{b} / \mathrm{w} \text { SAR } \\
\text { peaks }(\mathrm{D})(\mathrm{cm})\end{array}$ & $\begin{array}{l}\text { SPLSR } \\
=\frac{\left(S_{A R_{1}}+S A R_{2}\right)}{D} \\
(\mathrm{~W} / \mathrm{kg} / \mathrm{cm}\end{array}$ \\
\hline 0.77 & 0.35 & 0.46 & 2.9 & 0.28 & 0.12 & 0.19 & 2.6 & 0.12 \\
\hline 1.8 & 0.76 & 0.77 & 5.9 & 0.26 & 0.35 & 0.37 & 4.3 & 0.17 \\
\hline 2.1 & 0.52 & 0.35 & 4.2 & 0.21 & 0.42 & 0.23 & 3.9 & 0.16 \\
\hline
\end{tabular}

\section{CONCLUSIONS}

A small form factor and a low profile highly isolated multi-wideband MIMO antenna has been presented and investigated thoroughly. According to the surface current distribution over the antenna elements, neutralisation line, and the ground plane, it is depicted that the combination of neutralisation line and modified ground plane not only plays a vital role in isolation enhancement but also in impedance matching. The simulated and measured results of the proposed antenna confirms that the operating frequency bands are LTE700, GSM1800, GSM1900, UMTS, LTE2500, and Wi-Fi. The simulated and measured radiation patterns indicated that the proposed MIMO antenna system is a potential candidate for portable usages to combat multipath fading effect and promising for slim LTE/WWAN smart phone applications. The investigation of the proposed antenna in mobile environment and user proximity reasserted the use of the proposed antenna on actual scenario of the mobile phone by meeting the standard limit of SPLSR and TRP. The deep analysis of the proposed antenna in free space and user proximity makes it a suitable candidate for real time application in mobile handsets.

\section{REFERENCES}

Cellular Telecommunication Industry Association (CTIA) (2005). CTIA Certification Test Plan for Mobile Station Over the Air Performance. Method of Measurement for Radiated RF Power and Receiver Performance. CTIA Certification Program, Washington DC, USA.

Cellular Telecommunication Industry Association (CTIA) (2011). Test Plan for Mobile Station Over the Air Performance. CTIA Revision 3.1. CTIA Certification Program, Washington DC, USA.

Choukiker Y.K., Sharma S.K. \& Behera S.K. (2014). Hybrid fractal shape planar monopole antenna covering multiband wireless communications with MIMO implementation for handheld mobile devices. IEEE Transactions on Antennas Propagation 62(3): 1483-1488.

DOI: https://doi.org/10.1109/aps.2013.6710749

Coetzee J.C. \& Yu Y.T. (2008). Port decoupling for small arrays by means of an eigenmode feed network. IEEE Transactions on Antennas Propagation 56(6): 1587-1593. DOI: https://doi.org/10.1109/tap.2008.923301

Federal Communications Commission (FCC) (2008). SAR Evaluation Consideration for Handsets with Multiple Transmitters and Antennas. Federal Communications Commission, Washington DC, USA.

Gao Q., Mitra R., Lei F., Li Z., Ju J. \& Byun J. (2013). Interaction between internal antenna and external antenna of mobile phone and hand effect. IEEE Transactions on Antennas Propagation 61(2): 335-343. DOI: https://doi.org/10.1109/tap.2012.2220323

Meshram M.K., Animeh R.K., Pimpale A.T. \& Nikolova N.K. (2012). A novel quad-band diversity antenna for LTE and Wi-Fi applications with high isolation. IEEE Transactions on Antennas Propagation 60(9): 4360-4371.

DOI: https://doi.org/10.1109/tap.2012.2207044

Murch R.D. \& Letaief K.B. (2002). Antenna systems for broadband wireless access. IEEE Communication Magazine 40(4): 76-83.

DOI: https://doi.org/10.1109/35.995854

Pedersen K., Mogensen P. \& Fleury B. (1997). Power azimuth spectrum in outdoor environments. Electronics Letters 33(18): $1583-1584$.

DOI: https://doi.org/10.1049/el:19971029

Pelosi M., Franek O., Knudsen M.B., Pedersen G.F. \& Andersen J.B. (2010). Antenna proximity effects for talk and data modes in mobile phones. IEEE Antennas and Propagation Magazine 52(3): 15-26.

DOI: https://doi.org/10.1109/map.2010.5586570

Rowell C. \& Lam E.Y. (2012). Multiple frequency band and high isolation mobile device antennas using capacitive slot. IEEE Transactions on Antennas Propagation 60(8): 3576-3582.

DOI: https://doi.org/10.1109/tap.2012.2201077 
Singh H.S., Pandey G.K., Bharti P.K. \& Meshram M.K. (2015). Design and performance investigation of a low profile MIMO/Diversity antenna for WLAN/WiMAX/ HIPERLAN applications with high isolation. International Journal of RF and Microwave Computer Aided Engineering 25(6): 510-521.

DOI: https://doi.org/10.1002/mmce.20886

Singh H.S., Upadhyay R. \& Shubair R.M. (2018). Free space and user proximity analysis of octaband monopole $\mathrm{MIMO} /$ diversity antenna for modern handset applications. International Journal of $R F$ and Microwave Computer Aided Engineering 29(10): 1-13.

DOI: https://doi.org/10.1002/mmce.21566

Singh H.S., Upadhyay R. \& Shubair R.M. (2019). Performances study of compact printed diversity antennain the presence of user's body for LTE mobile phone applications. International Journal of $R F$ and Microwave Computer Aided Engineering 30(5): 1-11.

DOI: https://doi.org/10.1002/mmce. 21743

Singh H.S. \& Shubair R.M. (2019). User proximity analysis of compact PIFA for MIMO applications. URSI Radio Science Meeting-Antenna Propagation Society, 7-12 July, Atlanta, USA, pp. 33-34.

DOI: https://doi.org/10.1109/usnc-ursi.2019.8861710

Taga T. (1990). Analysis for mean effective gain of mobile antennas in land mobile radio environments. IEEE
Transactions on Vehicular Technology 39(2): 117-131 DOI: https://doi.org/10.1109/vetecf.2002.1040526

Vaughan R.G. \& Andersen J.B. (1987). Antenna diversity in mobile communications. IEEE Transactions on Vehicular Technology 36(4): 149-172. DOI: https://doi.org/10.1109/T-VT.1987.24115

Wong K.L., Kang T.W. \& Tu M.F. (2011). Internal mobile phone antenna array for LTE/WWAN and LTE MIMO operations. Microwave and Optical Technology Letters 53(7): 1569-1573.

DOI: https://doi.org/10.1002/mop.26038

Yu W., Yang S., Tang C.L. \& Tu D. (2010). Accurate simulation of the radiation performance of a mobile slide phone in a hand-head position. IEEE Antennas and Propagation Magazine 52(2): 168-177.

DOI: https://doi.org/10.1109/map.2010.5525613

Zhang S., Lau B.K., Sunesson A. \& He S. (2012). Closelypacked UWB MIMO/diversity antenna with differen patterns and polarizations for USB dongle application. IEEE Transactions on Antennas Propagation 60(9): 4372-4380.

DOI: https://doi.org/10.1109/tap.2012.2207049

Zhang S., Zhao K., Ying Z. \& He S. (2013). Adaptive quadelement multi-wideband antenna array for user-effective LTE MIMO mobile terminals. IEEE Transactions on Antennas Propagation 61(8): 4275-4283. DOI: https://doi.org/10.1109/tap.2013.2260714 